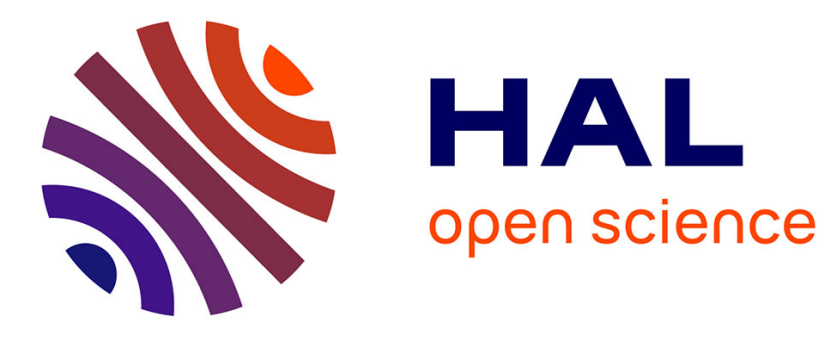

\title{
Water-like structure with repulsive double-core interactions
}

\author{
Aurélien Perera, Larisa Zoranic, Redha Mazighi, Franjo Sokolic
}

\section{To cite this version:}

Aurélien Perera, Larisa Zoranic, Redha Mazighi, Franjo Sokolic. Water-like structure with repulsive double-core interactions. Molecular Physics, 2009, 107 (13), pp.1349-1353. 10.1080/00268970902877787 . hal-00513282

\section{HAL Id: hal-00513282 \\ https://hal.science/hal-00513282}

Submitted on 1 Sep 2010

HAL is a multi-disciplinary open access archive for the deposit and dissemination of scientific research documents, whether they are published or not. The documents may come from teaching and research institutions in France or abroad, or from public or private research centers.
L'archive ouverte pluridisciplinaire HAL, est destinée au dépôt et à la diffusion de documents scientifiques de niveau recherche, publiés ou non, émanant des établissements d'enseignement et de recherche français ou étrangers, des laboratoires publics ou privés. 


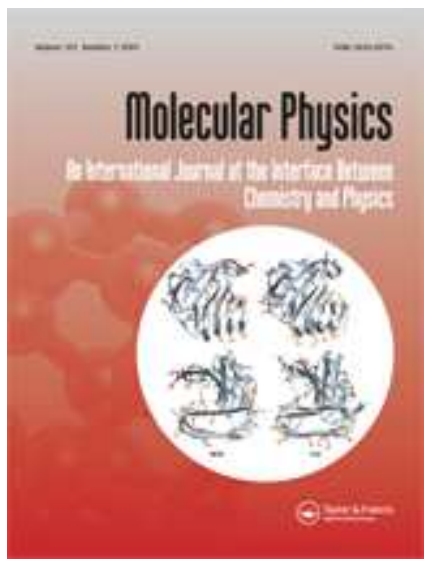

\section{Water-like structure with repulsive double-core interactions}

\begin{tabular}{|r|l|}
\hline Journal: & Molecular Physics \\
\hline Manuscript ID: & TMPH-2009-0058.R1 \\
\hline Manuscript Type: & Full Paper \\
\hline Author: & 06-Mar-2009 \\
\hline Complete List of Authors: & $\begin{array}{l}\text { Perera, Aurélien; Université Pierre et Marie Curie, Lab Physique } \\
\text { Théorique de la Matière Condensée } \\
\text { ZORANIC, Larisa; Université Pierre et Marie Curie, Physique } \\
\text { Théorique de la Matière Condensée } \\
\text { MAZIGHI, Redha; Université Pierre et Marie Curie, Physique } \\
\text { Théorique de la Matière Condensée } \\
\text { SOKOLIC, Franjo; Faculty of Sciences, University of Split, } \\
\text { Department of Physics }\end{array}$ \\
\hline Keywords: & water, liquid structure, liquid theory \\
\hline Note: The following files were submitted by the author for peer review, but cannot be converted \\
to PDF. You must view these files (e.g. movies) online.
\end{tabular}

\section{scholarONE" \\ Manuscript Central}




\title{
Water-like structure with repulsive
}

\section{double-core interactions}

\author{
Aurélien Perera ${ }^{1}$, Arnaud Rispe ${ }^{1}$, Larisa Zoranic ${ }^{1,2}$, Redha Mazighi ${ }^{1}$ and \\ Franjo Sokolic ${ }^{2}$
}

March 6, 2009

\author{
${ }^{1}$ Laboratoire de Physique Théorique de la Matière Condensée (UMR CNRS \\ 7600), Université Pierre et Marie Curie, 4 Place Jussieu, F75252, Paris \\ cedex 05, France. \\ ${ }^{2}$ Department of Physics, Faculty of Sciences, University of Split, Nikole \\ Tesle 12, 21000, Split, Croatia.
}

\begin{abstract}
The soft-core $1 / r^{12}$ repulsive interaction together with a Gaussian repulsive interaction are used to reproduce major features of the structure of liquid water, both in direct and reciprocal space, by Monte Carlo and integral equation theories. The study reveals that the structure of liquid water is determined, within the model studied here, by the competition of the two repulsive cores, which results in a two-fold
\end{abstract}


spatial distribution, very reminiscent of the two-state water model proposed by many authors. The fact is that many of the structural features of water could be reproduced without any recourse to direct attractive interactions, such as directional hydrogen bonds, and appear to be the result of long range competing packing correlations, as witnessed by the particular features of the structure factor. The Hypernetted-Chain integral equation is able to reproduce very accurately the most important features of the experimental structure of room temperature water, while the Percus-Yevick approximation fails to reach this state point. A high temperature study shows that this failure is related to the insufficiant diagrammatic structure of this closure.

\section{Introduction}

Water is an ubiquitous form of matter on earth. It is probably the substance that has the most known anomalous properties[1]. The properties of water are believed to stem directly from the hydrogen bonding (Hbond) interactions, which are then a key for its understanding[1]. This interaction has a quantum mechanical origin, the delocalisation of the hydrogen proton along the O-H..O direction. This interaction is then highly directional, and leads to the strong tetrahedrality of the ordering of neighbouring water molecules in all the thermodynamic phases. It turns out that relating the properties of water to the Hbond interactions by the use of statistical mechanics is an outstanding problem. Classical water force field models are numerous and none seems entirely adequate[2]. Many approaches has tried to explain the 
properties of water, such as the two-state models $[3,4,5]$ and the percolation model[6], for example. The experiments do not allow to decide in favour of either models[7]. From the statistical mechanical point of view, the structure of the liquid water holds partially the key to understanding its thermodynamical properties. The question is then, do we understand the features in the radial distribution function $(\mathrm{RDF})$ of water?

The RDF of water is well known from scattering experiments[8]. This structure is quite well reproduced by many classical models of water, such as the SPC models[9] and the TIPnP[10, 11] models, for example. Many refinements allow to fit minute details, but the essential features are already captured by these models. The very peculiar structure the RDF of water have been pointed out recently by one of us[12]. Statistical mechanical theory of liquids indicates that, if the structure of a liquid is accurately described, then the pair distribution alone would allow to reproduce most of its thermodynamical properties. This quantity will be then the focus of the present study, in particular through the integral equation formalism that allows to calculate this quantity directly from the pair interaction.

The fact that the RDF of water could be captured by a spherically symmetric interaction has been shown by Head-Gordon and Stillinger[13], who inverted the experimental RDF using integral equation closures. Very recently, other type of spherical models $[14,16]$ have been intensely used to understand the thermodynamical anomalies of water, mostly in the super cooled region[19]. All these type of interactions have one similarity in common, since they exhibit some sort of core-softening: in addition to the molecular size, another length scale is introduced through a second repulsive core. 
The existence of a second soft core appear sufficient to induce water-like properties, even in describing solvation properties[17].

Integral equations succeed in describing many types of liquids, from simple ones, such as argon, to more complex charged or orientatationally ordered ones[18]. However, in the case of room temperature water,they fail to reproduce the local tetrahedral ordering at contact[14], because of their incomplete diagrammatic structure[18]. Indeed, these theories give 12 neighbours, like in an ordinary hard sphere liquid, instead of 4 for water. It is only in the high supercritical temperature regime that they are quantitatively correct, because the strong tetrahedral packing is smeared by thermal agitation. It is important to note that this 4 neighbours structure is due both to the direct Hbonding interactions at contact, as well as the correlations induced by this interaction. Indeed, if water has an extended tetrahedral network structure, it is due to correlation effects. By using a simple model with two repulsive cores, we show here that the micro-structure of water is the result of a long ranged two-fold packing structure, due to the competition between these two cores. The use of repulsive interactions in order to mimic the real ones in real water need not be viewed as contradictory. Indeed, as was suggested by Jagla[14, 15], the strongly attractive directional Hbond interactions contributes to deplete the immediate neighbour of the water molecule, leading to 4 neighbours instead of 12 . It is this depletion interaction that is modeled by a second repulsive core, which then represents an effective Hbond interaction. Many studies[21, 20] have explored several aspect of such type of peculiar interactions, but none so far have noticed that the analogy is very deep since even the RDF can be similar to that of real water. This is the 
principal subject of this report.

\section{Results}

Since we are only interested in the dense liquid, we will neglect the attractive part of the dispersive interactions and focus here on the purely repulsive pair interaction given by

$$
v(r)=4 \epsilon\left(\frac{\sigma}{r}\right)^{12}+\epsilon_{G} \exp \left(-\alpha_{G}\left(r-r_{G}\right)^{2}\right)
$$

where the second term allows to soften the sharp $r^{12}$ repulsion. We choose here $\epsilon_{G} / \epsilon=30, \alpha_{G}=3.7 / \sigma^{2}$ and $r_{G}=0.85 \sigma$. The reduced temperature is then defined as $T^{*}=k_{B} T / \epsilon$, where $k_{B}$ is the Boltzmann constant. In order to stay in the liquid side of the phase diagram we choose $T^{*}=1.0$ and the reduced density $\rho^{*}=(N / V) \sigma^{3}=0.8$. Since we intent to capture the main features of water structure, and not fit the RDF itself[13], the exact state parameters of liquid water are not required. This system is studied by constant N,V,T Monte Carlo (MC) simulations, with $\mathrm{N}=4000$ particles in order to have a good description of the long range correlations in the system. Appropriate statistics ensure that we obtain smooth and noiseless RDF, as shown in the figures. We equally use integral equation theories, namely the Percus-Yevick (PY) and Hypernetted-Chain (HNC) closure relations[18], in order to find what approximate theories can tell us about such a system. These two theories differ by the way they relate the pair interaction $v(r)$, the radial distribution function $g(r)$ and the direct correlation function $c(r)$ : 


$$
g(r)=\exp \left(-v(r) / k_{B} T+h(r)-c(r)\right) \mathrm{HNC}
$$

$$
g(r)=\exp \left(-v(r) / k_{B} T\right)(1+h(r)-c(r)) \mathrm{PY}
$$

where $h(r)=g(r)-1$. It is seen that the PY closure is the first term in the expansion of the correlation term in the exponential of the HNC closure. One therefore expects that the PY theory will be missing infinite sets of diagrams that could be important in the study of dense liquids. But, it can also be argued that, if the log of both relations is taken, which means that it is $c(r)$ that is expressed in terms of $h(r)$, then it is the HNC closure that is a linearized version of the PY closure. This implies that the lack of diagrams in the PY closure could be only apparent, and that compensation effects could palliate this deficiency. Since, for hard spheres, the PY closure gives better results than the HNC closure, this has led to think that, for hard core and short range interactions, PY is superior to the HNC closure[18].

Fig. 1 shows $g(r)$ from the two approximations, for the $1 / r^{12}$ interaction without the core softening Gaussian part, compared with the MC simulation results. It is seen that both approximations bracket the exact results, but it is apparent that the HNC approximation is better. This is also reflected in the structure factor $S(k)=1+\rho \int d \vec{r} \exp (i \vec{k} \cdot \vec{r}) h(r)$, shown in the upper inset. The lower inset shows the integrand $r^{2} h(r)$, magnifying the decay of the correlations at large $r$. One sees that the PY approximation overestimates the liquid-like packing structure, particularly in the long range part, while the HNC approximation underestimates it. This is in sharp contrast with what 
happens near the contact at $r / \sigma \approx 1$. Here, it is HNC that overestimates the correlations at contact by displacing them inward, while PY places this first peak slightly outwards. This means that HNC approximation allows particles to get slightly closer at contact than the PY approximation does. This is in line with the known fact that, for hard spheres, the HNC approximation exaggerates the correlations at contact[18].

Fig. 2 shows the correlations when the core-softening part is turned on. The corresponding pair interaction is shown in the lower inset together with the $r^{12}$ interaction, and clearly shows that the core-softening produces an additional Gaussian repulsion at contact. The RDF from HNC is in excellent agreement with that from the simulations. Comparing $g(r)$ from Fig.1 and Fig. 2 reveals an outstanding result: the influence of the Gaussian interaction is two fold, firstly it shifts the correlations inward and closer to contact, and secondly the oscillatory structure is severely damped beyond the first three peaks. In order to see this more clearly, we show again in dashed lines the RDF from Fig.1 without the Gaussian repulsion. The first point is surprising, since one expects the influence of an additional repulsion to have the opposite effect. In fact, this is exactly what happens, except that the repulsion selects two configurations, one with correlations close to contact and the other mediated by the Gaussian repulsion. As a result of this twofold competitive repulsion, the particles closer at contact (at $r=\sigma$ ) appear more correlated since they are "trapped" by the second repulsive part. This explains why the oscillations in the new $g(r)$ are in phase opposition with those of the natural packing (dashed curve) with period $\sigma$. However, the most important finding here is that these new correlations resemble very 
much those of liquid water, as can be seen from the top inset, where both the RDF from the SPC/E water model and from X-ray scattering are shown. The damping of the oscillatory structure is the result of the phase opposing oscillations at long range, which indicates that the two features compete even at long range.

This resemblance throws a new lighting on the correlations in liquid water: the four-fold contact at neighbour is not only a result of the Hbond interactions, but also of the packing correlations induced by the two fold contact distance, that of the oxygen-oxygen contact $(\approx 3 \AA)$ and that of the orientation-restricted Hbond contact which is shorter $(\approx 2 \AA)$. This conclusion can be further confirmed by looking at the structure factor in Fig.3. The structure factor is important because it reflects structures that are hidden in the long range part of the RDF. Usually, such features appear near $k=0$, as for critical phenomena, or between $k=0$ and $k_{m}=2 \pi / \sigma$, the latter which corresponds to the main peak observed in the structure factor. In associated liquids such as liquid alcohols, for example, the structure factor shows a prepeak at $k=2 \pi / \xi$, indicating the existence clusters of average size $\xi[18]$. In the case of water, the experimental oxygen-oxygen structure factor, shown in the upper inset, together with that from the SPC/E model, is very peculiar because the "pre-peak" is very close to the main peak, suggesting that water clusters, if any, are made of near neighbour, from dimers to pentamers, as often suggested in the literature[1]. This is not in line with the fact that water might be a percolated liquid through its Hbond network[6]. This contradiction has never been satisfactorily resolved in the past literature on water[8]. From what was said above, we now see that the split-peak of $S(k)$ is in 
fact a "double main peak", corresponding to the two packing structures, one at $r \approx 0.8 \sigma$ and the other at $r \approx 1.2 \sigma$, and this double packing structure is present throughout the whole liquid. This interpretation of the structure factor finally resolves the puzzling absence of specific water clusters, when compared to liquid alcohols, as was observed in Ref[18].

The present study equally reveals that the PY theory is incapable of reaching the present structural state. The numerical solution is lost before the full Gaussian core is turned on. The reason of this failure is found by a high temperature study at $T^{*}=10$, with and without the Gaussian core, summarized in Fig.4. The corresponding interactions are shown in the lower inset. The upper inset shows the RDF without the Gaussian core, and the good agreement between both theories and the MC results. The packing structure is weak, since it is smeared by the higher thermal agitation. However, as the Gaussian core is turned on, whereas HNC continues to show weak structuring, in excellent agreement with MC simulations, PY shows very strong packing ordering, which indicates that this theory sees only the increase of repulsion at contact. The resulting increased packing structure explains why the correlations of this theory moves in the opposite direction than the real ones. This is a profound flaw of this theory, and reveals that this theory lacks the diagrams that are incorporated in the HNC theory, and not the other way around.

It is interesting to compare the methodology of our calculation to that of Head-Gordon and Stillinger[13]. When inverting the experimental RDF, which is exact, by using the HNC or PY closures, what is obtained is not the the spherical interaction itself, but rather the effective interaction affected 
by the missing structural diagrams. In the case of $\mathrm{HNC}$, for example, what is obtained is $v_{\text {eff }}(r)=v(r)-B(r)$, where $B(r)$ is the set of bridge diagrams. Therefore, some correlations get mixed in the final result. Needless to say, such interaction will vary with the density in a non-trivial way. The approach we use allows instead to start from a strict Hamiltonian definition of the interaction. The advantage is that diagramatic properties of integral equations, and in particular their ability to obtain the particular local structure of water, can be unambiguously captured.

\section{Conclusion}

This study reveals that water has an associating structure richer than that of simple alcohols, since it does not produce a local clustering, rather it spreads across the whole liquid. Hence water behaves like a giant polymer, as was suggested by Y. Koga on the basis of purely thermocalorimetric measurements[19]. It is interesting to note that the dual structure of water was hinted in the very first models of water[3, 5], but has always remained elusive since neither experiments nor computer simulations could provide a convincing evidence of such feature. In fact, this structure is difficult to observe unambiguously in snapshots, even in the supercooled regime[20]. But the $\mathrm{RDF}$ is the best tool to measure it. In this way, the RDF is the order parameter for the micro-structure of matter, just like the one-body function is the order parameter of the global order in Landau theory of phase transitions. The unexpected difference between the two most accurate theories that describe liquids, namely the PY and HNC approximations, indicate 
that a better progress can be achieved only by the explicit incorporation of higher forms of diagrams. These should allow a better description of the local clustering, that cannot obviously be reached by schemes that interpolate between these two closures[18]. The success of the current form of interaction that reproduces the behaviour of water indicates that missing diagrams can be modeled by such an effective repulsive interaction. We are currently exploring such pathways.

\section{References}

[1] F. Franks, Water, a comprehensive treatise, ( Plenum press, New York, 1972)

[2] B. Guillot, J. Mol. Liq. 101, 219 (2002).

[3] W. K. Roentgen, Ann. Phys. U. Chim. (Wied) 45 (1892) 91-97.

[4] L. Pauling, The structure of water, In Hydrogen bonding, Ed. D. Hadzi and H. W. Thompson (Pergamon Press Ltd, London, 1959)

[5] H. S. Frank and A. S. Quist, J. Chem. Phys. 34, 604 (1961)

[6] H. E. Stanley et al. J. Phys. Cond. Mat. 12, A403 (2000)

[7] H. J. Bakker, Chem. Rev. 108, 1456 (2008).

[8] T. Head-Gordon and H. Hura, Chem. Rev. 102, 2651 (2002)

[9] J. C. Berendsen, J.P.M. Postma, W.F. Von Gusteren and J. Hermans, in Intermolecular Forces, edited by B. Pullman (Reidel, Dortrecht, 1981). 
[10] W.L. Jorgensen, J. Chandrasekhar, J.D. Madura, R.W. Impey and M.L. Klein, J. Chem. Phys. 79, 926 (1983).

[11] M.W. Mahoney and W. L. Jorgensen, J. Chem. Phys. 112, 8910 (2000).

[12] A. Perera, submitted.

[13] T. Head-Gordon and F. Stillinger, J. Chem. Phys. 98, 3313 (1993)

[14] E. A. Yagla, Phys. Rev. E58, 1478 (1998)

[15] E. A. Yagla, J. Chem. Phys. 111, 8980 (1999)

[16] iG. Franzese, G. Malescio, A. Skibinsky, S. V. Buldyrev and H. E. Stanley, Nature 409, 692 (2001).

[17] S. V. Buldyrev, P. Kumar, P. G. Debenedetti, P. Rossky and H. E. Stanley, PNAS 104, 20117 (2007)

[18] J.P. Hansen and I.R. McDonald, Theory of Simple Liquids (Academic, London, 1986).

[14] J. Richardi, C. Millot and P. H. Fries, J. Chem. Phys. 110, 1138 (1999)

[19] O. Mishima and H. E. Stanley, Nature 396, 329 (1998)

[20] A. B. de Oliveira, G. Francese, P. A. Netz and M. C. Barbosa, J. Chem. Phys. 128, 064901 (2008)

[21] E. Lomba, N. G. Almarza, C. Martin and C. McBride, J. Chem. Phys. 126, $244510(2007)$

[18] L. Zoranić, F. Sokolić and A. Perera, J. Chem. Phys. 127, 324502 (2007) 
[19] Y. Koga, J. Phys. Chem. 100, 5172 (1996).

[20] J. Errington, P. G. Debenedetti and S. Torquato, Phys. Rev. Lett. 89, 215503-1 (2002)

13

14

15

16

17

18

19

20

21

22

23

24

25

26

27

28

29

30

31

32

33

34

35

36

37

38

39

40

41

42

43

44

45

46

47

48

49

50

51

52

53

54

55

56

57

58

59

60 


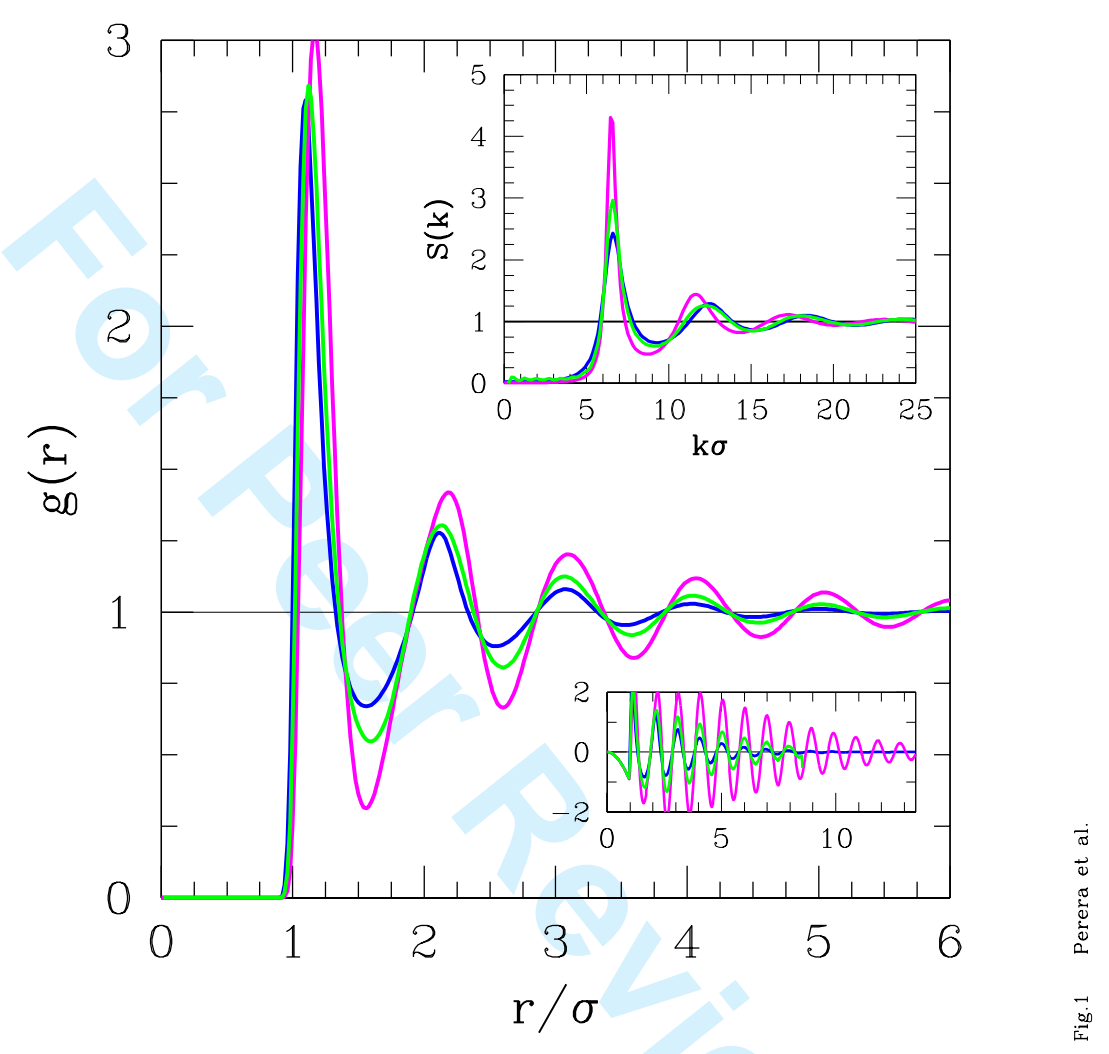

Fig.1 (color on line) The RDF for $1 / r^{12}$ interaction at state point $T^{*}=1$ and $\rho^{*}=0.8$. Blue curve for HNC, magenta for PY and green for MC. Lower inset: $r^{2} h(r)$ versus $r / \sigma$. Upper inset: structure factor. 


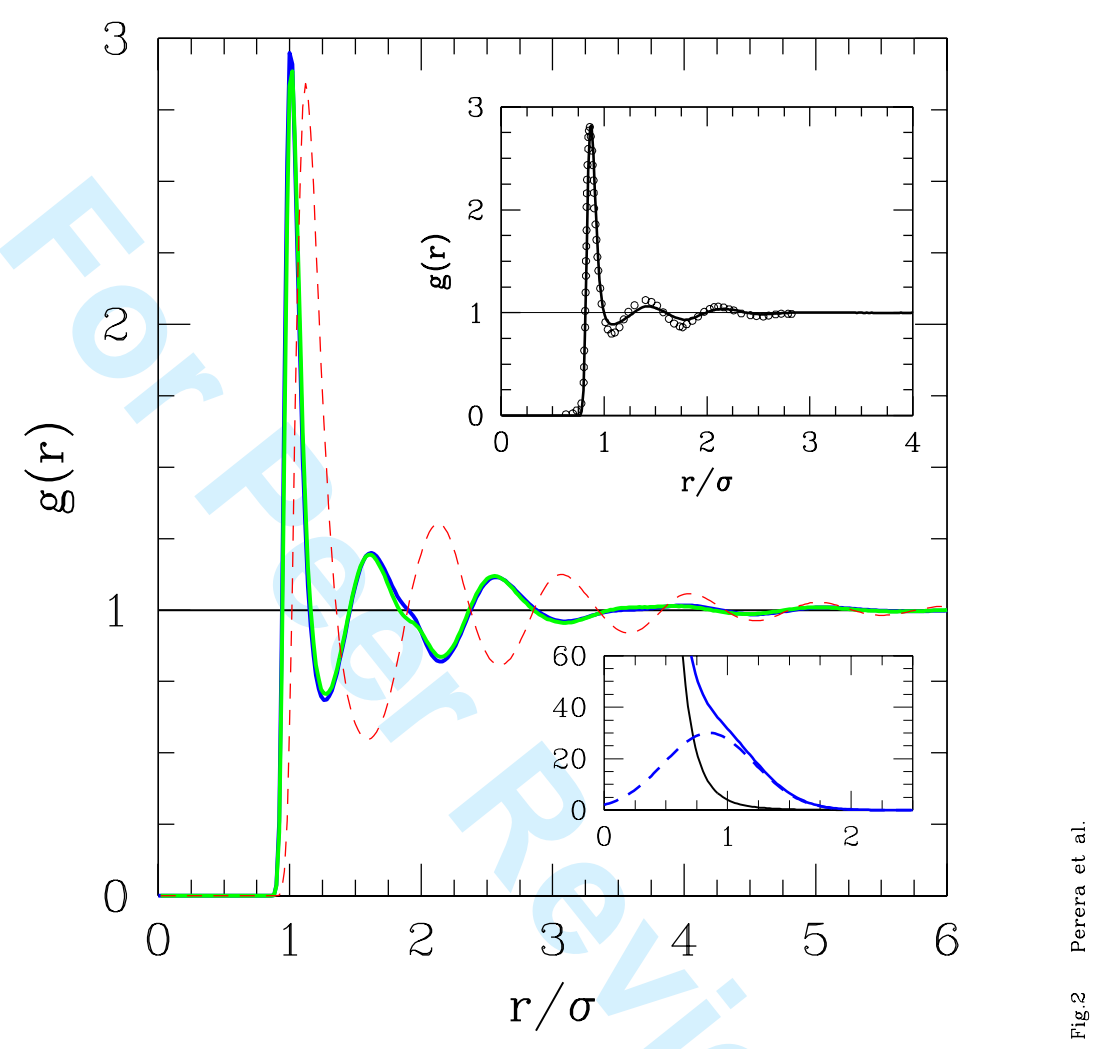

Fig.2 (color on line) The RDF for the full core-softened interaction for the same state point at in Fig.1 (with same color convention: PY results cannot be obtained for this case). The dashed curve in red is the MC RDF from Fig.1. Lower inset: the $1 / r^{12}$ (black), the gaussian repulsion (dashes) and the full (blue) interactions. Upper inset: RDF of neat water (dots SANS results from Ref., line SPC/E water model). 


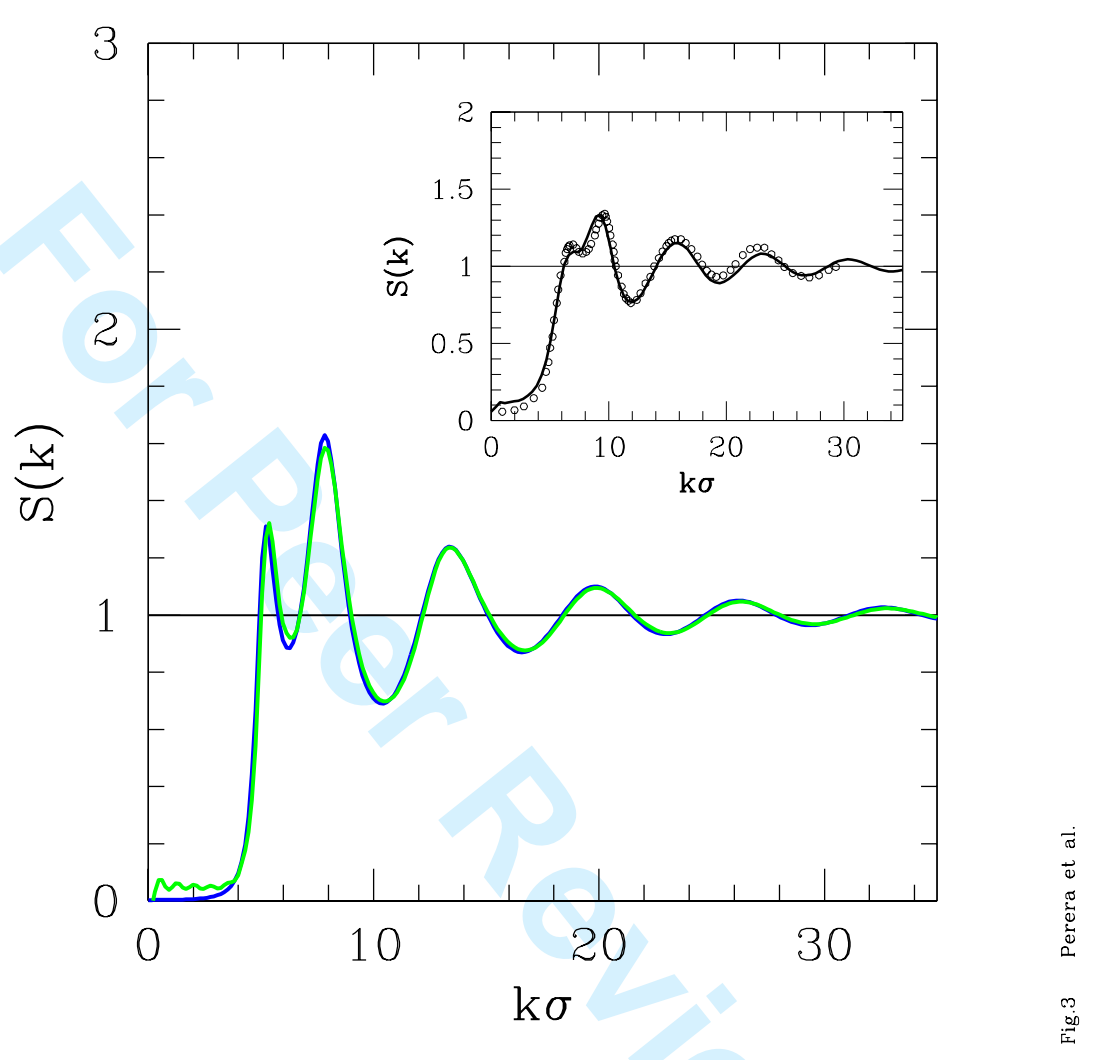

Fig.3 (color on line) Structure factors corresponding to the RDFs in Fig.2. The inset shows the experimental structure factor Ref. and that of the $\mathrm{SPC} / \mathrm{E}$ model. 


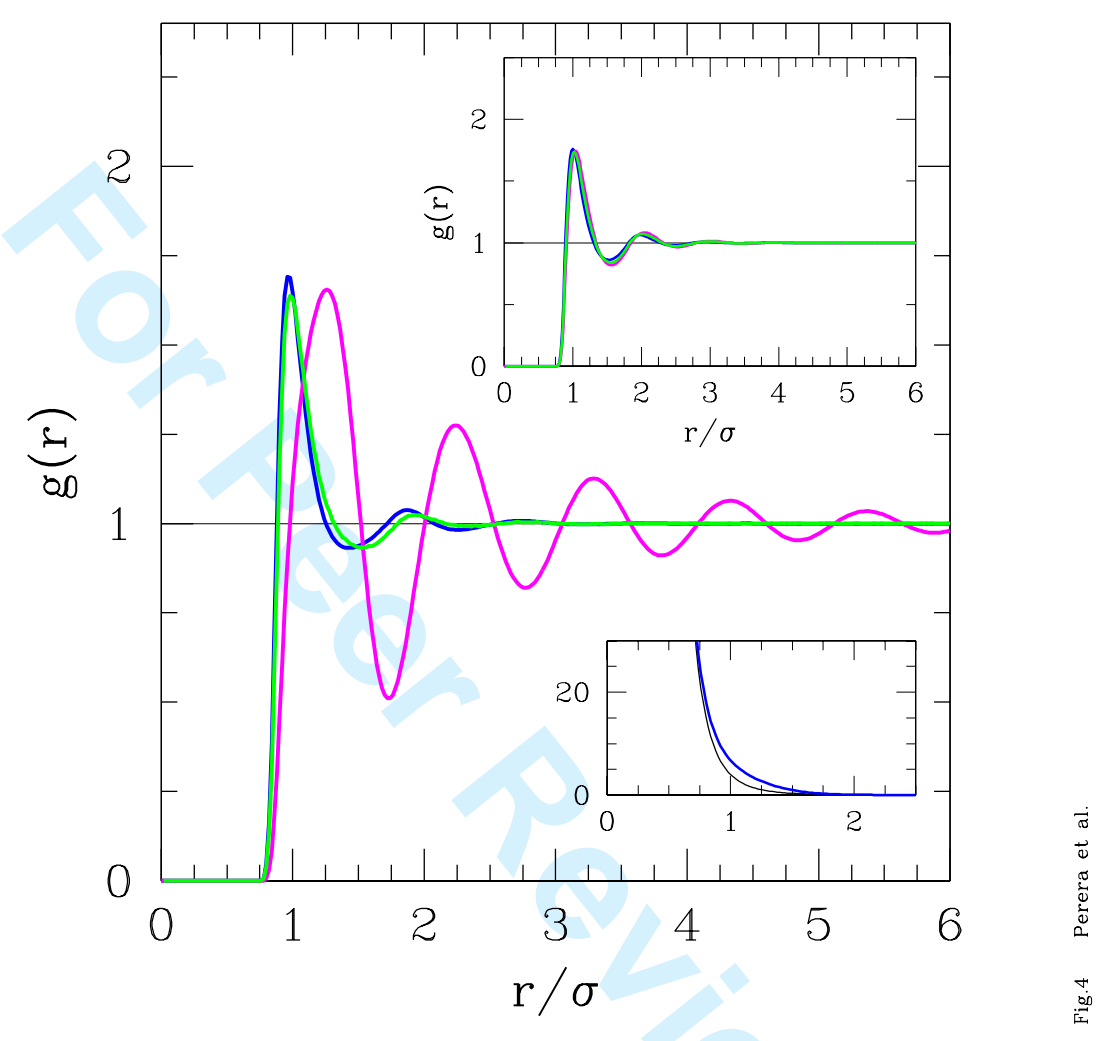

Fig.4 (color on line) RDF of the full core-soft interaction for high temperature $T^{*}=10$. Color conventions as in Fig.1. Lower inset: the pair interactions. Upper inset: the RDFs without the Gaussian core part. 
Reply to the Referee's comments on manuscript TMPH-2009-0058

We thank the Referee for his nice and constructive comments on our report. The manuscript have been revised, and in particular, equations are now numbered and we apologize for Ref.[13] which is now correctly displayed. The remaining points are addressed below.

1 ) Following his suggestion, we have added a new text (in blue in the pdf) in the introduction where we explain the connection between the Gaussian repulsion and the Hbond induced depletion which gives a number of neighbours lower than 12 .

2 ) We do not calculate three-body correlation functions in our MC simulations, only the pair correlations. There is no reasons to consider higher order correlation functions, and in particular the three-body ones, since there is no hint to what to expect at that level. The Referee seems to suggests that this function will "emphasize the competing packing structures more clearly", but it is not obvious to us how this goal will be reached by showing a function that will depend now on 3 variables (the two distances and the angle between them), and why this one in particular. We expect that all many body correlations will have contributions to the particular geometry, but it is not obvious how to analyze this in clear fashion. We are not aware that other authors have particularly illuminated the structure of water by showing such high order correlation functions. So, in absence of any specific information from the Referee, we are not sure how such calculation will enhance the present report. We will therefore stick to the pair correlations, which has the advantage over the higher order ones to be a true observable (through various scattering experiments).

3 ) The interaction in Eq.(1) was already displayed in the lower insert in Fig.2, where both the reference soft-core and the full interaction were shown. This was mentioned in the text where Fig.2 is quoted as well as in the Figure caption. We have added now the Gaussian repulsion in blue dashed line.

A part from the point 2), that we have not fully understood, we have answered to the Referees suggestions in the revised manuscript. 